\title{
A MATHEMATICAL MODEL FOR THE CONTROL AND ERADICATION OF A WOOD BORING BEETLE INFESTATION*
}

\author{
STEPHEN A. GOURLEY ${ }^{\dagger}$ AND XINGFU ZOU $¥$
}

\begin{abstract}
We propose a mathematical model for an infestation of a wooded area by a beetle species in which the larva develop deep in the wood of living trees. Due to the difficulties of detection, we presume that only a certain proportion of infested trees will be detected and that detection, if it happens, will occur only after some delay, which could be long. An infested tree once detected is immediately cut down and burned. The model is stage structured and contains a second time delay, which is the development time of the beetle from egg to adult. There is a delicate interplay between the two time delays due to the possibility in one case for a larva to mature even in a tree destined for destruction. We present conditions sufficient for infestation eradication and discuss the significance of the conditions, particularly in terms of the proportion of infested trees that need to be detected and removed. If the infestation is successfully eradicated, there are always a number of trees that completely escape infestation, and we compute lower bounds and an approximation for this number. Finally, we present the results of some numerical simulations.
\end{abstract}

Key words. delay, age-structure, infestation, eradication

AMS subject classifications. 34K25, 34K60, 92D30

DOI. $10.1137 / 060674387$

1. Introduction. In this paper we present a mathematical model of a possible strategy for the control of an infestation of wood boring beetles in which the larvae are burrowed deep in the wood of trees so that they are well protected from natural enemies but still have some intrinsic death rate. Our model also incorporates removal of trees that have been diagnosed as infested. Our work has been motivated in large part by recent infestations in parts of the US and Canada of Anoplophora glabripennis, commonly known as the Asian longhorned beetle (ALB), which attacks hardwood trees. Maple, willow, and elm constitute especially good hosts. Birch, ash, poplar, and numerous other tree species can also host this pest. The ALB has been intercepted at ports and warehouses all over North America, and it is believed that the pest entered the US (later spreading to Canada) in wooden packing crates used for imports from China. The beetle is native to China and Korea, and in China has caused major damage to poplar plantations with significant economic loss [6]. So far in North America the pest has affected only urban and suburban areas, but the potential impact of the ALB on the millions of acres of hardwood forests in the US and Canada could be devastating. It has been estimated that 1.2 billion trees could be at risk if the ALB were to become established in North America (see Nowak et al. [13] for a further discussion of the potential impact). Since the ALB species is not native to North America, it has no known natural enemies there (in fact, even in China it has few natural enemies).

${ }^{*}$ Received by the editors November 8, 2006; accepted for publication (in revised form) March 17, 2008; published electronically June 25, 2008. This research was partially supported by NSERC and NCE-MITACS of Canada and by PREA of Ontario.

http://www.siam.org/journals/siap/68-6/67438.html

${ }^{\dagger}$ Department of Mathematics, University of Surrey, Guildford, Surrey GU2 7XH, UK (s.gourley@ surrey.ac.uk).

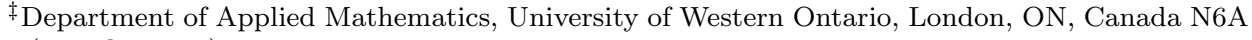
5B7 (xzou@uwo.ca).

1665 
The first time the ALB was found in North America seems to have been in Brooklyn, NY in 1996. Since then, the species has been found infesting trees in several US cities, including New York and Chicago, and it was found in Toronto, Canada for the first time in 2003.

Currently, the only known effective method of control of the ALB is to cut down, chip, and burn infested trees. Chemical controls are of limited potential because the larvae are deep within the tree and no effective chemical controls are yet available, though the effectiveness of certain insecticides is being investigated. It is current practice to establish quarantines around known infested areas and to monitor potential host trees within a certain distance of an infested area. Adult beetles are poor fliers but can fly short distances up to a few hundred yards to other neighboring trees, though in fact an adult often remains on the same host tree from which it emerged. ALB infested trees once removed are always replaced with a nonhost species. Other measures currently used for control include inspection of imports and the imposition of regulations on wooden packing material used for imports.

The ALB is a large beetle (up to 1.5 in long) which is easy to recognize. Adults are active from late spring to fall, when they perish. However, a large proportion of the life cycle of the beetle is spent in the larval stage deep within a tree, and this makes the detection of ALB activity more difficult. ALB larval activity on a tree is usually spotted either by inspectors or by members of the public. Warning signs of a tree being infested include exit holes (typically the diameter of a dime), oozing sap, sawdust accumulation, and unseasonable yellowing or drooping of leaves. The females prefer to lay their eggs in the upper canopy of a tree, though the lower trunk and branches can become affected if the upper canopy has been damaged by previous ALB activity. Preference for the upper canopy means that detection is more likely if inspectors are able to inspect it, for example, by climbing the tree. This slows down and increases the expense of systematically searching for ALB in a wooded area with the consequence of trees potentially missing detection. We shall aim to include these factors in the models we present in this paper. Since at present ALB affects only urban or suburban areas of North America, an infested tree probably stands a reasonable chance of being diagnosed as such, though possibly not until some time after the laying of eggs. An infested tree, if detected, will always be cut down and burned, but if not detected, the tree may survive several more years. Its death in this case will be due to the weakening of the tree and disruption of sap flow caused by the tunneling due to the larvae.

Adult ALB of both sexes are promiscuous, mating repeatedly and with different partners, according to greenhouse experiments reported in Morewood et al. [11]. The female will chew through the bark on the upper trunk and lay an egg. A single female can lay from 35 to 90 eggs during her lifetime of one season. The egg hatches after 1-2 weeks and the larva burrows deep into the tree, where it is very well protected from natural enemies, so there is a high probability of survival to maturity (this probability does depend to some extent on the tree species; see Morewood et al. [12]). Later in its development the larva enters the pupa stage, and finally the adult emerges from the tree. The whole duration from egg to adult lasts about one year but can be as long as 18 months. Adult emergence creates visible exit holes, which can sometimes be seen with binoculars, though the holes are not the only or necessarily the earliest sign of tree infestation.

In our models we shall deal with the issue of infested tree detection by supposing that there is a time delay $\sigma$ between the time a tree becomes infested and the subsequent detection of ALB activity on the tree. To allow for the difficulties of 
detection we assume that only a certain fraction $\lambda$ of infested trees is detected and then immediately removed. Such a fraction might well be close to 1 , if the infestation is confined to a small suburban wooded area, but is likely to be much smaller if ALB infestation were to develop in a wilderness area. The models include a second time delay $\tau$ which models the developmental time of the beetle from egg to adult. As we shall see, there is a delicate interplay between the two delays $\sigma$ and $\tau$ for reasons that will be explained early in the next section.

Subsection 2.1 deals with the case when $\sigma<\tau$ and presents the model for this case together with a detailed derivation. Positivity of solutions is established, which is not at all obvious from the appearance of the model equations. Then, sufficient conditions are presented for infestation eradication and a lower bound is given for the final number of susceptible trees.

Subsection 2.2 addresses the case $\sigma>\tau$. The model equations for this case look similar to those for the $\sigma<\tau$ case, but there are subtle differences, and a different strategy is required to establish positivity of solutions. For this case we again present an inequality that is sufficient for infestation eradication. Subsection 2.3 deals with the case when $\lambda=1$. In subsection 2.4 we present a Laplace transform analysis that enables us to calculate analytically the final number of susceptible trees in the case when the number of adult beetles is small throughout the course of the infestation.

In some parameter regimes the infestation is not eradicated but instead all trees become infested, with the number of susceptible trees tending to zero (we shall see, however, that if the infestation is eradicated, there are always some trees that escape infestation). In situations in which eradication has not been achieved, the beetle numbers typically evolve to a periodic cycle in a forest in which all trees end up being infested. In reality the goal, of course, is to prevent this from happening and aim for eradication, but without necessarily requiring the detection and removal of every single infested tree.

2. Model derivation and analysis. Let $T_{s}(t)$ and $T_{i}(t)$ denote, respectively, the numbers of susceptible and infested trees. Trees can survive about 4 years of infestation before they die; this is somewhat longer than the timescale on which we would want to remove infested trees, so disease-induced death of infested trees is neglected. It is also reasonable to neglect natural mortality of trees which occurs on an even longer timescale (e.g., of 100 years or more for maple trees). The quantities $L(t)$ and $A(t)$ denote the numbers of larval and adult beetles.

The model we shall develop involves two time delays. We shall let $\sigma$ denote the amount of time that elapses between the instant that a tree becomes infested and the subsequent instant at which there is a probability $\lambda$ of its being removed and burnt as a consequence (i.e., a fraction $\lambda \in[0,1]$ of trees that become infested are removed $\sigma$ time units later). We shall let $\tau$ denote the time it takes between the laying of an egg and subsequent emergence of an adult beetle, i.e., the duration of the larval stage, which in this paper is understood to include all pre-adult stages. It will be clear that the cases $\sigma<\tau$ and $\sigma>\tau$ have to be dealt with separately. For example, if $\sigma<\tau$, then the period between time of infection of a tree and its subsequent removal (if it is removed) is not long enough to allow any larva to mature; however, a larva can still mature if it is fortunate enough to be in a host tree that is not removed. On the other hand if $\sigma>\tau$, then it is possible for larvae to complete their development into maturity and escape as adult beetles even if all infested trees are removed. 
2.1. The case $\sigma<\tau$. For the case when $\sigma<\tau$, we propose the following model:

$$
\frac{d T_{s}(t)}{d t}=-\beta A(t) T_{s}(t)
$$

$$
\frac{d T_{i}(t)}{d t}=\beta A(t) T_{s}(t)-\lambda \beta A(t-\sigma) T_{s}(t-\sigma),
$$

$$
\begin{aligned}
& \frac{d L(t)}{d t}=T_{i}(t) B(A(t))-\mu_{L} L(t)-\lambda \beta A(t-\sigma) T_{s}(t-\sigma) \int_{0}^{\sigma} B(A(t-a)) e^{-\mu_{L} a} d a \\
& -e^{-\mu_{L} \tau} B(A(t-\tau))\left[T_{i}(t-\tau)-\lambda \beta \int_{0}^{\sigma} A(\tilde{a}+t-\tau-\sigma) T_{s}(\tilde{a}+t-\tau-\sigma) d \tilde{a}\right]
\end{aligned}
$$

$$
\begin{aligned}
\frac{d A(t)}{d t}= & e^{-\mu_{L} \tau} B(A(t-\tau))\left[T_{i}(t-\tau)-\lambda \beta \int_{0}^{\sigma} A(\tilde{a}+t-\tau-\sigma) T_{s}(\tilde{a}+t-\tau-\sigma) d \tilde{a}\right] \\
& -\mu_{A} A(t) .
\end{aligned}
$$

Here, all parameters are nonnegative with $\lambda \in[0,1]$. We justify each equation in (2.1)(2.4) below.

Susceptible trees are converted to infested trees via contact with adult beetles, and it is assumed that the rate at which this occurs is given by the law of mass action (equation (2.1)). There is no term reflecting regeneration of trees, partly because this would occur on a relatively slow timescale and partly because tree replanting would be of some nonsusceptible species and might not take place at all while the infestation is still present.

The second term in the right-hand side of (2.2) represents the cutting down (and subsequent burning) of infested trees. It is assumed that when a tree becomes infested, it may be recognized and diagnosed as such but only after some time delay $\sigma$, which models the time taken for the tree to begin exhibiting telltale signs. A fraction $\lambda \in[0,1]$ of trees which become infested are later cut down, so that at time $t$ the rate of cutting down of infested trees is $\lambda$ times the infection rate at the earlier time $t-\sigma$.

The first term in the right-hand side of (2.3) is the birth rate, assumed proportional to the total number of infested hosts (recall that a tree is considered infested after contact with an adult beetle) and also to $B(A(t))$, where the function $B(\cdot)$ is the number of eggs laid per unit time per tree. We assume that all eggs hatch successfully, but some of the larvae may die in the tree at a rate $\mu_{L}$. Of course, larvae may also die due to trees being cut down and burned. The rate at which this happens is evidently related to the total cutting down rate of infested and dead trees and is computed as follows:

$$
\underbrace{\lambda \beta A(t-\sigma) T_{s}(t-\sigma)}_{\text {rate of tree removal }} \underbrace{\int_{0}^{\sigma} B(A(t-a)) e^{-\mu_{L} a} d a}_{\text {larvae per tree }} .
$$

The last term in (2.3), which also appears in (2.4), is the rate at time $t$ at which larvae mature into adult beetles. We next provide a rigorous derivation of this term, which is essentially the birth rate at the earlier time $t-\tau$ ( $\tau$ being the length of the 
maturation period), modified to allow for natural mortality and mortality due to tree removal. Death of adult beetles is modeled by the last term in (2.4).

To derive the maturation term for the case when $\sigma<\tau$, let $b(t, a)$ denote the density of beetles at time $t$ of age $a$. Larval beetles and adult beetles are, respectively, those of age less than $\tau$ and greater than $\tau$ so that

$$
L(t)=\int_{0}^{\tau} b(t, a) d a, \quad A(t)=\int_{\tau}^{\infty} b(t, a) d a .
$$

It is larval beetles that are affected by removal of trees, but we must note that, since we assume $\sigma<\tau$ here, the larvae that are removed due to tree removal will have age up to at most $\sigma$. Any older larvae will necessarily be in trees that escaped removal. We model this as follows using von Foerster age-structured equations:

$$
\begin{gathered}
\frac{\partial b}{\partial t}+\frac{\partial b}{\partial a}=-\mu_{L} b(t, a)-\lambda \beta A(t-\sigma) T_{s}(t-\sigma) B(A(t-a)) e^{-\mu_{L} a}, \quad a<\sigma \\
\frac{\partial b}{\partial t}+\frac{\partial b}{\partial a}=-\mu_{L} b(t, a), \quad \sigma<a<\tau .
\end{gathered}
$$

The explanation for the last term in the right-hand side of (2.6) is as follows. It is the rate at which larvae of age $a$ are removed due to tree removal, and is therefore the rate of tree removal $\lambda \beta A(t-\sigma) T_{s}(t-\sigma)$, times the larvae density of age $a$ per tree that is thus removed, which will be the birth rate per tree at time $t-a$ times the probability of survival to age $a$, i.e., $B(A(t-a)) e^{-\mu_{L} a}$.

For adult beetles,

$$
\frac{\partial b}{\partial t}+\frac{\partial b}{\partial a}=-\mu_{A} b(t, a), \quad a>\tau .
$$

Differentiating the expression for $A(t)$ in (2.5) gives

$$
\frac{d A}{d t}=b(t, \tau)-\mu_{A} A,
$$

assuming that $b(t, \infty)=0$. We shall find $b(t, \tau)$ in terms of the birth rate $b(t, 0)$ by integrating (2.6) and (2.7) along characteristics. Since we previously defined $B(A(t))$ as the number of eggs laid per unit time per tree, the birth rate $b(t, 0)$ is given by

$$
b(t, 0)=T_{i}(t) B(A(t)) .
$$

Define

$$
b_{\zeta}(a)=b(a+\zeta, a)
$$

Then, for $a \leq \sigma$,

$$
\begin{aligned}
\frac{d b_{\zeta}(a)}{d a} & =\left[\frac{\partial b}{\partial t}+\frac{\partial b}{\partial a}\right]_{t=a+\zeta} \\
& =-\mu_{L} b_{\zeta}(a)-\lambda \beta A(a+\zeta-\sigma) T_{s}(a+\zeta-\sigma) B(A(\zeta)) e^{-\mu_{L} a} .
\end{aligned}
$$

Solving this for $b_{\zeta}(a)$ leads to

$b(t, a)=$

$e^{-\mu_{L} a} B(A(t-a))\left[T_{i}(t-a)-\lambda \beta \int_{0}^{a} A(\tilde{a}+t-a-\sigma) T_{s}(\tilde{a}+t-a-\sigma) d \tilde{a}\right], \quad a \leq \sigma$. 
For ages $a$ between $\sigma$ and $\tau$, an easier calculation involving (2.7) shows that

$$
b(t, a)=b(t-(a-\sigma), \sigma) e^{-\mu_{L}(a-\sigma)},
$$

and $b(t-(a-\sigma), \sigma)$ can be found from (2.10) giving that, for $\sigma \leq a \leq \tau$,

$$
b(t, a)=e^{-\mu_{L} a} B(A(t-a))\left[T_{i}(t-a)-\lambda \beta \int_{0}^{\sigma} A(\tilde{a}+t-a-\sigma) T_{s}(\tilde{a}+t-a-\sigma) d \tilde{a}\right] .
$$

This expression looks rather like the corresponding one for $a \leq \sigma$ (expression (2.10)), but note that the upper limit on the integral is now $\sigma$ rather than $a$. This difference is very important. Putting $a=\tau$ into (2.11) gives an expression for $b(t, \tau)$, which we insert into (2.9), thereby completing the derivation of (2.4).

The expression for $b(t, \tau)$ is those larvae of age $\tau$ and represents the rate at which larval beetles become adult beetles (the adult recruitment rate). Expression (2.11) with $a=\tau$ shows that this is basically the birth rate at the earlier time $t-\tau$ (corrected for larval mortality) minus those larvae that would have made it to adulthood but were removed and destroyed with their host tree (the integral term represents accumulated removal of trees that could have hosted the larvae we are discussing, i.e., trees that became infested at times between $t-\tau-\sigma$ and $t-\tau)$. An alternative viewpoint is that the term in square brackets in (2.11) (with $a=\tau$ ) is the "effective" number of host trees at time $t-\tau$ when the eggs are laid since, in this $\sigma<\tau$ regime, those trees that are removed might as well not have been there in the first place.

The derivation of the larval equation (2.3) is by differentiation of the expression for $L(t)$ in (2.5), breaking the integral up into the $a<\sigma$ and $a \in(\sigma, \tau)$ contributions, and using (2.6) and (2.7).

As we are considering the $\sigma<\tau$ situation, in which it is impossible for a larva to complete its development in a tree that gets removed, we should expect that when $\lambda=1$ (i.e., every tree that becomes infested is later removed), the maturation rate $b(t, \tau)$ should be zero. When (and only when) $\lambda=1$, the number of infested trees at time $t$ is given by

$$
T_{i}(t)=\int_{t-\sigma}^{t} \beta A(\xi) T_{s}(\xi) d \xi
$$

Therefore, indeed, $b(t, \tau)=0$ when $\lambda=1$, for $\sigma<\tau$.

2.1.1. Initial data and positivity. The initial data for system (2.1), (2.2), (2.3), (2.4) has the form

$$
\begin{aligned}
T_{s}(t)= & T_{s}^{0}(t) \geq 0, \quad t \in[-\tau-\sigma, 0], \\
A(t)= & A^{0}(t) \geq 0, \quad t \in[-\tau-\sigma, 0], \\
T_{i}(t)= & \beta \int_{-\sigma}^{0} A^{0}(t+\xi) T_{s}^{0}(t+\xi) d \xi, \quad t \in[-\tau, 0], \\
L(0)= & \int_{-\tau}^{0} B\left(A^{0}(\xi)\right) e^{\mu_{L} \xi} T_{i}(\xi) d \xi \\
& -\lambda \beta \int_{0}^{\sigma} \int_{-\tau}^{-a} B\left(A^{0}(\xi)\right) A^{0}(a+\xi-\sigma) T_{s}^{0}(a+\xi-\sigma) e^{\mu_{L} \xi} d \xi d a,
\end{aligned}
$$

where $T_{s}^{0}(t)$ and $A^{0}(t)$ are prescribed continuous functions. The last two conditions in (2.12) are compatibility conditions, by which we mean that the initial data for $T_{i}$ 
and $L$ is not arbitrary but is computed from the prescribed nonnegative initial data for $T_{s}$ and $A$. For example, the larvae present at time $t=0$ are the offspring of the adults at earlier times, and the modeling described thus far in this paper leads to the expression in (2.12) for $L(0)$. The initial data (2.12) including the compatibility conditions is the only ecologically relevant initial data.

Proposition 1. Let $\sigma<\tau$ and $\lambda \in[0,1]$, and let the initial data for system (2.1), (2.2), (2.3), (2.4) satisfy (2.12). Let the birth function be bounded and satisfy $B(0)=0$ and $B(A)>0$ for $A>0$. Then all variables in (2.1)-(2.4) are defined for all $t>0$ and are bounded and remain nonnegative for $t>0$.

Proof. Existence of solutions follows by the method of steps which, since $\sigma<\tau$ here, is carried out successively on the steps $t \in[0, \sigma], t \in[\sigma, 2 \sigma]$, etc. It is easily seen that this works on the subsystem consisting of (2.1), (2.2), and (2.4). For example when $t \in[\sigma, 2 \sigma]$, all arguments of the delayed variables in (2.4) remain less than $\sigma$. So, local existence is assured for $T_{s}(t), T_{i}(t)$, and $A(t)$ (global existence will be shown later). It turns out that $L(t)$ has an explicit expression in terms of these variables (see (2.17) below). Next we shall show that all variables remain nonnegative for as long as they are defined.

It is obvious that $T_{s}(t) \geq 0$ for all $t>0$. Next we shall show nonnegativity of $A(t)$. This will be achieved by jointly showing nonnegativity of $A(t)$ and the function $f(t)$ defined by

$$
f(t)=T_{i}(t)-\lambda \beta \int_{t-\sigma}^{t} A(\xi) T_{s}(\xi) d \xi .
$$

Using (2.2) we find that

$$
\frac{d f}{d t}=\beta(1-\lambda) A(t) T_{s}(t)
$$

Also, (2.4) can be rewritten in a form involving $f(t)$,

$$
\frac{d A(t)}{d t}=e^{-\mu_{L} \tau} B(A(t-\tau)) f(t-\tau)-\mu_{A} A(t) .
$$

As regards initial conditions for $f(t)$, note that when $t \in[-\tau, 0]$, from (2.12),

$$
\begin{aligned}
f(t) & =\beta \int_{-\sigma}^{0} A(t+\xi) T_{s}(t+\xi) d \xi-\lambda \beta \int_{t-\sigma}^{t} A(\xi) T_{s}(\xi) d \xi \\
& =(1-\lambda) \beta \int_{-\sigma}^{0} A(t+\xi) T_{s}(t+\xi) d \xi \geq 0
\end{aligned}
$$

so $f(t)$ is nonnegative initially. The functions $f(t)$ and $A(t)$ can be viewed as satisfying (2.14) and (2.15), considered here as a coupled system in which $T_{s}(t)$ is some known nonnegative function. The assumptions on $B(\cdot)$ and the nonnegativity of $f(t)$ and $A(t)$ for $t \leq 0$ allow us to deduce, from Theorem 2.1 on page 81 of Smith [16], that $f(t) \geq 0$ and $A(t) \geq 0$ for all $t>0$.

From (2.2),

$$
\begin{aligned}
\frac{d T_{i}(t)}{d t} & =\beta A(t) T_{s}(t)-\lambda \beta A(t-\sigma) T_{s}(t-\sigma) \\
& \geq \beta A(t) T_{s}(t)-\beta A(t-\sigma) T_{s}(t-\sigma)=\frac{d}{d t} \int_{t-\sigma}^{t} \beta A(\xi) T_{s}(\xi) d \xi
\end{aligned}
$$

Copyright $@$ by SIAM. Unauthorized reproduction of this article is prohibited. 
Thus $T_{i}(t)-\int_{t-\sigma}^{t} \beta A(\xi) T_{s}(\xi) d \xi$ is an increasing function of $t$ which, by (2.12), is zero when $t=0$. Hence

$$
T_{i}(t) \geq \int_{t-\sigma}^{t} \beta A(\xi) T_{s}(\xi) d \xi \geq 0
$$

for $t>0$.

Finally, we address positivity of $L(t)$. The solution of (2.3) subject to the compatibility condition in (2.12) is most easily found from (2.5) with (2.10) and (2.11) and turns out to be

$$
\begin{aligned}
L(t)= & \int_{t-\tau}^{t} B(A(\xi)) e^{-\mu_{L}(t-\xi)} T_{i}(\xi) d \xi \\
& -\lambda \beta \int_{0}^{\sigma} \int_{t-\tau}^{t-a} B(A(\xi)) A(a+\xi-\sigma) T_{s}(a+\xi-\sigma) e^{-\mu_{L}(t-\xi)} d \xi d a .
\end{aligned}
$$

Indeed, the most general solution of (2.3), which is linear in $L(t)$, is the above expression plus $C \exp \left(-\mu_{L} t\right)$ for some constant $C$, and the latter term would have to be set to zero to satisfy (2.12). The state space of initial data in (2.12) is forward invariant in this sense.

Using (2.13) and nonnegativity of $f(t)$, it can be shown that

$$
L(t) \geq \int_{0}^{\sigma} \int_{t-\bar{\xi}}^{t} B(A(\xi)) A(\bar{\xi}+\xi-\sigma) T_{s}(\bar{\xi}+\xi-\sigma) e^{-\mu_{L}(t-\xi)} d \xi d \bar{\xi} .
$$

Hence $L(t) \geq 0$. Having shown nonnegativity of each solution variable while it is defined, we may now establish global existence. This can be done by establishing a priori bounds. Indeed, nonnegativity of the variables and (2.1) imply that $T_{s}(t)$ is decreasing, so that $T_{s}(t)$ is bounded above and below. Since $d f / d T_{s}=-(1-\lambda)$, it follows that $f(t)$ is also bounded above and below. It then follows from (2.15), using boundedness of $B(\cdot)$, that $A(t)$ is bounded. Then (2.13) implies that $T_{i}(t)$ is bounded. Then expression (2.17) shows that $L(t)$ is bounded and the proof is complete.

2.1.2. Infestation eradication. In this section we present conditions on the parameters which guarantee that for initial data satisfying (2.12) in section 2.1.1 the infestation is eradicated.

TheOREM 1. Let $\sigma<\tau$ and $\lambda \in[0,1]$, and let the initial data for system (2.1), (2.2), (2.3), (2.4) satisfy (2.12). Let the birth function satisfy $B(0)=0$ and $0<B(A) \leq B^{\prime}(0) A$ for $A>0$. Assume further that

$$
(1-\lambda) e^{-\mu_{L} \tau} B^{\prime}(0)\left(T_{s}(0)+\beta \int_{-\sigma}^{0} A^{0}(\xi) T_{s}^{0}(\xi) d \xi\right)<\mu_{A} .
$$

Then the solution of the system satisfies $A(t) \rightarrow 0$ and $L(t) \rightarrow 0$ as $t \rightarrow \infty$, so that the infestation is eradicated.

Furthermore, the final number $T_{s}(\infty)$ of susceptible trees is not less than

$$
T_{s}(0) \exp \left(-\beta\left\{\frac{A^{0}(0)+(1-\lambda) e^{-\mu_{L} \tau} B^{\prime}(0)\left(T_{s}(0)+\beta \int_{-\sigma}^{0} A^{0}(\xi) T_{s}^{0}(\xi) d \xi\right) \int_{-\tau}^{0} A^{0}(\xi) d \xi}{\mu_{A}-(1-\lambda) e^{-\mu_{L} \tau} B^{\prime}(0)\left(T_{s}(0)+\beta \int_{-\sigma}^{0} A^{0}(\xi) T_{s}^{0}(\xi) d \xi\right)}\right\}\right) .
$$


Remark 1. Inequality (2.18) essentially arises from the worst imaginable (but not actually attainable) scenario in which the entire forest becomes infested before the infestation is eradicated. If the infestation is successfully eradicated, there will always be some trees that escape infestation, and (2.19) gives a lower bound for this number of escaped trees. However, in the proof of the theorem we are faced with the difficulty that the number $T_{s}(\infty)$ is not known exactly. We need an upper bound for the function $f(t)$ defined by (2.13) involving only known quantities, and in achieving this we are forced to use $T_{s}(\infty) \geq 0$ so that we are effectively considering an extreme but unattained situation. The quantity $T_{s}(0)+\beta \int_{-\sigma}^{0} A^{0}(\xi) T_{s}^{0}(\xi) d \xi$ is the total initial number of trees (susceptible and infested), all of which would end up infested in this worst case scenario. But a fraction $1-\lambda$ of them is not removed. The left-hand side of (2.18) is the per capita maturation rate at large times, being the per capita egg laying rate per tree $B^{\prime}(0)$, multiplied by the number of infested trees at large times corrected for tree removal, multiplied by the survival probability $e^{-\mu_{L} \tau}$.

Proof of Theorem 1. From (2.1) and nonnegativity of solutions, $T_{s}(t)$ is a decreasing nonnegative function which therefore approaches a nonnegative limit as $t \rightarrow \infty$.

Recall the function $f(t)$ defined by (2.13). From (2.1) and (2.14) note that

$$
\frac{d f}{d T_{s}}=-(1-\lambda)
$$

Hence

$$
f(t)=-(1-\lambda) T_{s}(t)+T_{i}(0)-\lambda \beta \int_{-\sigma}^{0} A^{0}(\xi) T_{s}^{0}(\xi) d \xi+(1-\lambda) T_{s}(0) .
$$

Since $T_{s}(t) \geq 0$

$$
\begin{aligned}
f(t) & \leq T_{i}(0)-\lambda \beta \int_{-\sigma}^{0} A^{0}(\xi) T_{s}^{0}(\xi) d \xi+(1-\lambda) T_{s}(0) \\
& =(1-\lambda)\left[T_{s}(0)+\beta \int_{-\sigma}^{0} A^{0}(\xi) T_{s}^{0}(\xi) d \xi\right] \quad \text { using (2.12). }
\end{aligned}
$$

Using the form of (2.4) involving $f(t)$ (i.e., (2.15)) and the above upper bound for $f(t)$, we obtain

$$
\begin{aligned}
\frac{d A(t)}{d t} & \leq(1-\lambda) e^{-\mu_{L} \tau} B(A(t-\tau))\left(T_{s}(0)+\beta \int_{-\sigma}^{0} A^{0}(\xi) T_{s}^{0}(\xi) d \xi\right)-\mu_{A} A(t) \\
& \leq(1-\lambda) e^{-\mu_{L} \tau} B^{\prime}(0) A(t-\tau)\left(T_{s}(0)+\beta \int_{-\sigma}^{0} A^{0}(\xi) T_{s}^{0}(\xi) d \xi\right)-\mu_{A} A(t) .
\end{aligned}
$$

Since the right-hand side of this is increasing as a function of the delayed variable $A(t-\tau)$, we may say that $A(t)$ is bounded above by the solution of the differential equation obtained by replacing " $\leq$ " by "=" and satisfying the same initial data as that for $A$ (see, e.g., Theorem 1.1 on page 78 of Smith [16]). By a straightforward and standard argument involving the characteristic equation of the resulting linear delay equation, utilizing Theorem 5.1 on page 92 of Smith [16] to assure ourselves that the dominant eigenvalue is real, we conclude that $A(t) \rightarrow 0$ as $t \rightarrow \infty$ under the hypothesis (2.18). The proof that $L(t) \rightarrow 0$ follows from (2.3) and the theory 
of asymptotically autonomous systems (see, e.g., Castillo-Chavez and Thieme [2]). Furthermore since the convergence of $A(t)$ to zero will be exponential, we are assured that $\int_{0}^{\infty} A(t) d t<\infty$, which is necessary for what follows.

Integrating (2.21) from 0 to $\infty$ and rearranging gives

$$
\int_{0}^{\infty} A(t) d t \leq \frac{A^{0}(0)+(1-\lambda) e^{-\mu_{L} \tau} B^{\prime}(0)\left(T_{s}(0)+\beta \int_{-\sigma}^{0} A^{0}(\xi) T_{s}^{0}(\xi) d \xi\right) \int_{-\tau}^{0} A^{0}(\xi) d \xi}{\mu_{A}-(1-\lambda) e^{-\mu_{L} \tau} B^{\prime}(0)\left(T_{s}(0)+\beta \int_{-\sigma}^{0} A^{0}(\xi) T_{s}^{0}(\xi) d \xi\right)} .
$$

Inserting this estimate into

$$
T_{s}(\infty)=T_{s}(0) \exp \left(-\beta \int_{0}^{\infty} A(t) d t\right),
$$

which follows from (2.1), gives the estimate (2.19). The proof is complete.

2.2. The case $\sigma>\tau$. If $\sigma>\tau$, then the maturation time for a larva is less than the time that elapses between a tree becoming infested and its possible subsequent removal $\sigma$ time units later. Thus, if an egg is laid on a particular tree just after that tree became infested, then that larva is not at risk of having its host tree removed and burned. If an egg is laid on a tree that became infested some time ago, such that the tree now has less than $\tau$ time units to go before the time at which there is a probability $\lambda$ of its being removed, that larva could still survive to maturation if its host is not actually removed. These considerations lead us to the following different model equations:

$$
\begin{aligned}
& \frac{d L(t)}{d t}=T_{i}(t) B(A(t))-\mu_{L} L(t)-\lambda \beta A(t-\sigma) T_{s}(t-\sigma) \int_{0}^{\tau} B(A(t-a)) e^{-\mu_{L} a} d a \\
& -e^{-\mu_{L} \tau} B(A(t-\tau))\left[T_{i}(t-\tau)-\lambda \beta \int_{0}^{\tau} A(\tilde{a}+t-\tau-\sigma) T_{s}(\tilde{a}+t-\tau-\sigma) d \tilde{a}\right]
\end{aligned}
$$

$$
\begin{aligned}
\frac{d A(t)}{d t}= & e^{-\mu_{L} \tau} B(A(t-\tau))\left[T_{i}(t-\tau)-\lambda \beta \int_{0}^{\tau} A(\tilde{a}+t-\tau-\sigma) T_{s}(\tilde{a}+t-\tau-\sigma) d \tilde{a}\right] \\
& -\mu_{A} A(t) .
\end{aligned}
$$

All parameters are again nonnegative with $\lambda \in[0,1]$.

This system looks very similar to the corresponding system for $\sigma<\tau$ described in subsection 2.1, but there is an important difference: the upper limits in the integrals in (2.23) and (2.24) are $\tau$ rather than $\sigma$. This is because in this case, we need only break down $b(t, a)$ into two cases: (i) when $a<\tau, b(t, a)$ is governed by the PDE in (2.6); and (ii) for $a>\tau, b(t, a)$ is governed by the PDE in (2.8). The derivation of (2.23) and (2.24) is similar to but even simpler than that of (2.3) and (2.4).

Copyright $@$ by SIAM. Unauthorized reproduction of this article is prohibited. 
The first goal for this model is again to prove positivity of the solutions corresponding to the initial compatibility conditions (2.12). First, (2.21) gives $T_{s}(t)=$ $T_{s}(0) \exp \left(-\beta \int_{0}^{t} A(\theta) d \theta\right)>0$ for all $t \geq 0$. In order to obtain the positivity of other variables, we introduce a new variable,

$$
\begin{aligned}
g(t) & =T_{i}(t)-\lambda \beta \int_{0}^{\tau} A(\tilde{a}+t-\sigma) T_{s}(\tilde{a}+t-\sigma) d \tilde{a} \\
& =T_{i}(t)-\lambda \beta \int_{t-\sigma}^{t+\tau-\sigma} A(\xi) T_{s}(\xi) d \xi
\end{aligned}
$$

Then we have

$$
\frac{d g(t)}{d t}=\beta A(t) T_{s}(t)-\lambda \beta A(t+\tau-\sigma) T_{s}(t+\tau-\sigma),
$$

and the adults equation (2.24) can be rewritten as

$$
\frac{d A(t)}{d t}=e^{-\mu_{L} \tau} B(A(t-\tau)) g(t-\tau)-\mu_{A} A(t) .
$$

Since $g(t)$ does not behave as nicely as the function $f(t)$ in subsection 2.1, we have to tackle positivity via another strategy.

Note that, for $t \in[0, \sigma-\tau]$,

$$
\frac{d g(t)}{d t} \geq \beta A(t) T_{s}(t)-\beta A(t-(\sigma-\tau)) T_{s}(t-(\sigma-\tau))=\frac{d}{d t} \int_{t-(\sigma-\tau)}^{t} \beta A(\xi) T_{s}(\xi) d \xi,
$$

implying that $g(t)-\int_{t-(\sigma-\tau)}^{t} \beta A(\xi) T_{s}(\xi) d \xi$ is increasing on $[0, \sigma-\tau]$. Thus, for $t \in[0, \sigma-\tau]$, we have

$$
\begin{aligned}
g(t) & -\int_{t-(\sigma-\tau)}^{t} \beta A(\xi) T_{s}(\xi) d \xi \\
& \geq g(0)-\int_{-(\sigma-\tau)}^{0} \beta A(\xi) T_{s}(\xi) d \xi \\
& =T_{i}(0)-\lambda \int_{-\sigma}^{-(\sigma-\tau)} \beta A(\xi) T_{s}(\xi) d \xi-\int_{-(\sigma-\tau)}^{0} \beta A(\xi) T_{s}(\xi) d \xi \\
& \geq T_{i}(0)-\int_{-\sigma}^{-(\sigma-\tau)} \beta A(\xi) T_{s}(\xi) d \xi-\int_{-(\sigma-\tau)}^{0} \beta A(\xi) T_{s}(\xi) d \xi \\
& =T_{i}(0)-\int_{-\sigma}^{0} \beta A(\xi) T_{s}(\xi) d \xi=0 .
\end{aligned}
$$

Hence,

$$
g(t) \geq \int_{t-(\sigma-\tau)}^{t} \beta A(\xi) T_{s}(\xi) d \xi \quad \text { for } \quad t \in[0, \sigma-\tau]
$$

This implies that if $A(0)>0$ and $T_{s}(0)>0$ (recalling that $A^{0}(\theta)$ and $T^{0}(\theta)$ are continuous in (2.12)), then $g(0)>0$. Let $\delta=\min \{\tau, \sigma-\tau\}$; then either both $A(t)$ and $g(t)$ remain positive on $[0, \delta]$ or $A(t)$ will become negative before $g(t)$. In the

Copyright (c) by SIAM. Unauthorized reproduction of this article is prohibited. 
latter case, there is a $t_{0} \in(0, \delta]$ such that $A\left(t_{0}\right)=0, A(t)>0$, and $g(t)>0$ for $t<t_{0}$. It follows from (2.27) that

$$
A^{\prime}\left(t_{0}\right)=e^{-\mu_{L} \tau} B\left(A\left(t_{0}-\tau\right)\right) g\left(t_{0}-\tau\right)>0,
$$

which is impossible. This contradiction shows that $A(t)>0$ and $g(t)>0$ for all $t \in[0, \delta]$. Repeating this process, we can obtain the positivity of $A(t)$ and $T_{s}(t)$ on $[0,2 \delta]$ and, by induction, on $[0, n \delta]$ for all positive integers $n$, giving the positivity of $A(t)$ and $T_{s}(t)$ for all $t>0$.

Once we have obtained the positivity of $A(t)$ and $T_{s}(t)$, the positivity of $T_{i}(t)$ and $L(t)$ can be obtained in precisely the same way as in subsection 2.1. Therefore, we have obtained the following positivity result for (2.21)-(2.24), parallel to Proposition 1 for (2.1)-(2.4).

Proposition 2. Let $\sigma>\tau$ and $\lambda \in[0,1]$, and let the initial data for system (2.21)-(2.24) satisfy $(2.12)$ with $A(0)>0$ and $T_{s}(0)>0$. Let the birth function satisfy $B(0)=0$ and $B(A)>0$ for $A>0$. Then all variables in (2.21)-(2.24) remain nonnegative for $t>0$.

We next seek conditions under which the infestation will be eradicated. Adding (2.21) and (2.26) gives

$$
\frac{d}{d t}\left[g(t)+T_{s}(t)\right]=-\lambda \beta A(t-(\sigma-\tau)) T_{s}(t-(\sigma-\tau)) \leq 0 .
$$

Thus,

$$
\begin{aligned}
g(t) & \leq g(t)+T_{s}(t) \leq g(0)+T_{s}(0) \\
& =T_{i}(0)-\lambda \beta \int_{-\sigma}^{-(\sigma-\tau)} A(\xi) T_{s}(\xi) d \xi+T_{s}(0) \\
& =\beta \int_{-\sigma}^{0} A(\xi) T_{s}(\xi) d \xi-\lambda \beta \int_{-\sigma}^{-(\sigma-\tau)} A(\xi) T_{s}(\xi) d \xi+T_{s}(0) \\
& =(1-\lambda) \beta \int_{-\sigma}^{-(\sigma-\tau)} A(\xi) T_{s}(\xi) d \xi+\beta \int_{-(\sigma-\tau)}^{0} A(\xi) T_{s}(\xi) d \xi+T_{s}(0) .
\end{aligned}
$$

Therefore, in the case $\sigma>\tau$, if (2.18) is replaced by

$e^{-\mu_{L} \tau} B^{\prime}(0)\left[(1-\lambda) \beta \int_{-\sigma}^{-(\sigma-\tau)} A(\xi) T_{s}(\xi) d \xi+\beta \int_{-(\sigma-\tau)}^{0} A(\xi) T_{s}(\xi) d \xi+T_{s}(0)\right]<\mu_{A}$,

then by an argument similar to that in the proof of Theorem 1, we can conclude that the solution of system (2.21)-(2.24) with the initial compatibility conditions (2.12) satisfies $A(t) \rightarrow 0$ and $L(t) \rightarrow 0$ as $t \rightarrow \infty$; that is, the infestation will be eradicated. We have proved the following theorem.

Theorem 2. Let $\sigma>\tau$ and $\lambda \in[0,1]$, and let the initial data for system (2.21), (2.22), (2.23), (2.24) satisfy $(2.12)$ with $T_{s}(0)>0$ and $A(0)>0$. Let the birth function satisfy $B(0)=0$ and $0<B(A) \leq B^{\prime}(0) A$ for $A>0$. Assume further that (2.31) holds. Then the solution of the system satisfies $A(t) \rightarrow 0$ and $L(t) \rightarrow 0$ as $t \rightarrow \infty$ so that the infestation is eradicated.

As in section 2.1, under the assumptions in Theorem 2 and based on the $T_{s}(t)$ and $A(t)$ equations (i.e., (2.21) and (2.27)) and the estimate (2.30) for the function 
$g(t)$, we can also establish a lower bound for $T_{s}(\infty)$, the final number of susceptible trees. Indeed, if we denote by $M$ the right-hand side of (2.30), then by an argument similar to that for obtaining the estimate (2.19), we can derive the following lower bound for $T_{s}(\infty)$ :

$$
T_{s}(\infty) \geq T_{s}(0) \exp \left(-\beta\left\{\frac{A^{0}(0)+e^{-\mu_{L} \tau} B^{\prime}(0) M \int_{-\tau}^{0} A^{0}(\xi) d \xi}{\mu_{A}-e^{-\mu_{L} \tau} B^{\prime}(0) M}\right\}\right) .
$$

2.3. The case $\lambda=1$. In this subsection we discuss the situation when $\lambda=1$, which means that every tree that becomes infested is removed $\sigma$ time units later. We shall deal with both of the cases $\sigma<\tau$ and $\sigma>\tau$. In either case, the expression

$$
T_{i}(t)=\int_{t-\sigma}^{t} \beta A(\xi) T_{s}(\xi) d \xi
$$

is available to us, though the implications of this fact for the cases $\sigma<\tau$ and $\sigma>\tau$ are different. Expression (2.33) gives us the total number of infested trees at time $t$, which, when $\lambda=1$, is simply the accumulation of all new infestations over the previous $\sigma$ units of time (the corresponding expression for $T_{i}(t)$ when $\lambda \neq 1$ appears later (expression (2.39)) and in this case includes trees that became infested before time $t-\sigma$ but escaped detection).

In the case $\sigma<\tau$ the use of expression (2.33) in (2.4) yields $A^{\prime}(t)=-\mu_{A} A(t)$ so that $A(t) \rightarrow 0$, and the infestation is eradicated. This is hardly surprising since if every infested tree is removed $\sigma$ time units after the time of infestation, and $\sigma<\tau$, then no larva is being given enough time to mature.

If $\sigma>\tau$, the equation for $A(t)$ is (2.24), and the use of (2.33) leads to

$$
\frac{d A(t)}{d t}=e^{-\mu_{L} \tau} B(A(t-\tau)) \int_{t-\sigma}^{t-\tau} \beta A(\xi) T_{s}(\xi) d \xi-\mu_{A} A(t) .
$$

We will use this equation to show that the infestation is always eradicated when $\lambda=1$, regardless of the values of $\sigma$ and $\tau$. The truth of this result even in the $\sigma>\tau$ case is a little surprising, since this case offers the possibility of some larvae maturing before their host tree is destroyed. However, numerical simulations do show that even though eradication is still the final outcome, there may be a long transient in which the infestation grows worse for a while before dying out.

THEOREM 3. Let $\lambda=1$, and consider the system consisting of either (2.1)-(2.4) or (2.21)-(2.24), with initial data satisfying $(2.12)$ with $T_{s}(0)>0$ and $A(0)>0$. Let the birth function satisfy $B(0)=0$ and $0<B(A) \leq B^{\prime}(0) A$ for $A>0$. Then the infestation is eradicated, that is, $\left(T_{i}(t), L(t), A(t)\right) \rightarrow(0,0,0)$ as $t \rightarrow \infty$.

Proof. It is sufficient to show that $A(t) \rightarrow 0$. Then $L(t) \rightarrow 0$ follows trivially from (2.3) or (2.23) as appropriate, and $T_{i}(t) \rightarrow 0$ follows from (2.33). We have already commented above that if $\sigma<\tau$, then $A(t) \rightarrow 0$ trivially; this can be extended to $\sigma=\tau$. So it remains to consider the case $\sigma>\tau$, and it is here that we shall make use of (2.34), which has to be coupled to (2.21). The latter equation, together with nonnegativity of solutions, implies that $T_{s}(t)$ must decay monotonically to some nonnegative limit as $t \rightarrow \infty$. If $T_{s}(t) \rightarrow 0$, then the asymptotic limit of (2.34) is just $A^{\prime}(t)=-\mu_{A} A(t)$, and so $A(t) \rightarrow 0$. So it remains to consider the case that 
$\lim _{t \rightarrow \infty} T_{s}(t)>0$. If this is so, then from (2.21) it follows that

$$
\int_{0}^{\infty} A(t) d t<\infty
$$

If we can show that, additionally,

$$
\int_{0}^{\infty}\left|A^{\prime}(t)\right| d t<\infty
$$

then a result from integration theory assures us that $\lim _{t \rightarrow \infty} A(t)=0$. The integral in (2.34) can be expressed in terms of $T_{s}(t-\tau)$ and $T_{s}(t-\sigma)$ using (2.21), and the monotonicity properties of $T_{s}(t)$ therefore assure us of the existence of a finite $C$ such that

$$
\left|\int_{t-\sigma}^{t-\tau} \beta A(\xi) T_{s}(\xi) d \xi\right| \leq C \text { for all } t \geq 0
$$

Therefore, integration of (2.34) and using the estimate $0<B(A) \leq B^{\prime}(0) A$ lead to

$$
\int_{0}^{\infty}\left|A^{\prime}(t)\right| d t \leq C e^{-\mu_{L} \tau} B^{\prime}(0) \int_{-\tau}^{0} A^{0}(t) d t+\left(C e^{-\mu_{L} \tau} B^{\prime}(0)+\mu_{A}\right) \int_{0}^{\infty} A(t) d t .
$$

Hence $\int_{0}^{\infty}\left|A^{\prime}(t)\right| d t<\infty$. The proof is complete.

2.4. Approximation of $\boldsymbol{T}_{\boldsymbol{s}}(\boldsymbol{\infty})$. In subsections 2.1 and 2.2 we have seen that, under the eradication condition (2.18) for system (2.1)-(2.4), or condition (2.31) for system (2.21)-(2.24), the infestation is eradicated and the beetle does not affect the entire forest, since $A(t) \rightarrow 0$ and $T_{s}(t) \rightarrow T_{s}(\infty)=: T_{s}^{*}>0$. Lower bounds for $T_{s}^{*}$ are provided by inequalities (2.19) and (2.32) for models (2.1)-(2.4) and (2.21)-(2.24), respectively. In this subsection we provide an approach for obtaining an approximation for $T_{s}^{*}$. We present our analysis only for $(2.21)-(2.24)$; the analysis for $(2.1)-(2.4)$ is similar.

It is easily seen using (2.26) that the function

$$
t \rightarrow g(t)-\int_{t-(\sigma-\tau)}^{t} \beta A(\xi) T_{s}(\xi) d \xi
$$

is increasing for all $t \geq 0$. But (2.30) implies that this function is bounded from above. Therefore, it has a limit as $t \rightarrow \infty$, and thus $g(t)$ also has a limit as $t \rightarrow \infty$, since we consider the situation in which $A(t) \rightarrow 0$. It follows from (2.25) that $T_{i}^{*}:=$ $\lim _{t \rightarrow \infty} T_{i}(t)$ also exists and $T_{i}^{*}=\lim _{t \rightarrow \infty} g(t)$. Now, when $t$ is sufficiently large, $A(t)$ becomes very small, and hence we may study the linearized approximation of (2.27) for small $A(t)$, and also replace $g(t-\tau)$ by its (as yet undetermined) limiting value $T_{i}^{*}$ as $t \rightarrow \infty$ to obtain

$$
\frac{d A(t)}{d t}=e^{-\mu_{L} \tau} B^{\prime}(0) T_{i}^{*} A(t-\tau)-\mu_{A} A(t) .
$$

Letting $p$ be the transform variable, the Laplace transform $\bar{A}(p)$ of $A(t)$ is

$$
\bar{A}(p)=\frac{A^{0}(0)+e^{-\mu_{L} \tau} T_{i}^{*} B^{\prime}(0) e^{-p \tau} \int_{-\tau}^{0} A^{0}(\xi) e^{-p \xi} d \xi}{p-e^{-\mu_{L} \tau} T_{i}^{*} B^{\prime}(0) e^{-p \tau}+\mu_{A}} .
$$

Copyright $\odot$ by SIAM. Unauthorized reproduction of this article is prohibited. 
The structure of (2.37) assures us that the dominant eigenvalue of its characteristic equation is real (see Smith [16]). Furthermore, this dominant eigenvalue is negative, since we consider the situation when $A(t) \rightarrow 0$, so let it be $-p^{*}\left(T_{i}^{*}\right)$, to emphasize the dependence on $T_{i}^{*}$, with $p^{*}\left(T_{i}^{*}\right)>0$. This dominant eigenvalue is also the singularity of $\bar{A}(p)$ of greatest real part. By the inversion formula for Laplace transforms, $A(t)$ can be expressed as a contour integral, which by Cauchy's residue formula can be evaluated as a sum of residues of the poles of $\bar{A}(p)$. We shall include in this calculation only the pole of greatest real part, which is located at $p=-p^{*}\left(T_{i}^{*}\right)$, to give

$$
\begin{aligned}
A(t) & \approx \operatorname{res}\left(\bar{A}(p) e^{p t}, p=-p^{*}\left(T_{i}^{*}\right)\right) \\
& =\frac{e^{-p^{*}\left(T_{i}^{*}\right) t}\left[A^{0}(0)+e^{-\mu_{L} \tau} T_{i}^{*} B^{\prime}(0) e^{p^{*}\left(T_{i}^{*}\right) \tau} \int_{-\tau}^{0} A^{0}(\xi) e^{p^{*}\left(T_{i}^{*}\right) \xi} d \xi\right]}{1+\tau e^{-\mu_{L} \tau} T_{i}^{*} B^{\prime}(0) e^{p^{*}\left(T_{i}^{*}\right) \tau}} .
\end{aligned}
$$

Now, from (2.21),

$$
T_{s}^{*}=T_{s}(0) \exp \left(-\beta \int_{0}^{\infty} A(t) d t\right)
$$

giving

$$
T_{s}^{*} \approx T_{s}(0) \exp \left(-\frac{\beta\left[A^{0}(0)+e^{-\mu_{L} \tau} T_{i}^{*} B^{\prime}(0) e^{p^{*}\left(T_{i}^{*}\right) \tau} \int_{-\tau}^{0} A^{0}(\xi) e^{p^{*}\left(T_{i}^{*}\right) \xi} d \xi\right]}{p^{*}\left(T_{i}^{*}\right)\left(1+\tau e^{-\mu_{L} \tau} T_{i}^{*} B^{\prime}(0) e^{p^{*}\left(T_{i}^{*}\right) \tau}\right)}\right) .
$$

The solution of (2.22), subject to the initial value formula for $T_{i}(0)$ from $(2.12)$, is

$$
T_{i}(t)=\int_{t-\sigma}^{t} \beta A(\xi) T_{s}(\xi) d \xi+(1-\lambda) \int_{-\sigma}^{t-\sigma} \beta A(\xi) T_{s}(\xi) d \xi,
$$

which states that the number of infested trees at time $t$ is the accumulated total of newly infested trees since time $t-\sigma$, plus the accumulated total over all times prior to $t-\sigma$ that escaped being cut down. Since we consider the case when $A(t) \rightarrow 0$, we can use (2.39) to find $T_{i}^{*}$ in terms of $T_{s}^{*}$ as follows:

$$
\begin{aligned}
T_{i}^{*} & =(1-\lambda) \int_{-\sigma}^{\infty} \beta A(\xi) T_{s}(\xi) d \xi \\
& =(1-\lambda)\left[\int_{-\sigma}^{0} \beta A^{0}(\xi) T_{s}^{0}(\xi) d \xi-\int_{0}^{\infty} \frac{d T_{s}(\xi)}{d \xi} d \xi\right],
\end{aligned}
$$

giving

$$
T_{i}^{*}=(1-\lambda)\left[\int_{-\sigma}^{0} \beta A^{0}(\xi) T_{s}^{0}(\xi) d \xi+T_{s}(0)-T_{s}^{*}\right] .
$$

Recall that $p^{*}\left(T_{i}^{*}\right)>0$ has been defined such that $p=-p^{*}\left(T_{i}^{*}\right)$ is the singularity of $\bar{A}(p)$ of greatest real part. Therefore, $p^{*}\left(T_{i}^{*}\right)$ satisfies

$$
-p^{*}\left(T_{i}^{*}\right)-e^{-\mu_{L} \tau} T_{i}^{*} B^{\prime}(0) e^{p^{*}\left(T_{i}^{*}\right) \tau}+\mu_{A}=0 .
$$

Equation (2.41) defines $p^{*}\left(T_{i}^{*}\right)$ as a function of $T_{i}^{*}$, and then (2.38) and (2.40) are solved simultaneously for $T_{s}^{*}$ and $T_{i}^{*}$.

Copyright $@$ by SIAM. Unauthorized reproduction of this article is prohibited. 
3. Numerical simulations. We have carried out some numerical simulations of our model for both the $\sigma\langle\tau$ and $\sigma\rangle \tau$ situations. Fortunately, the alternative formulations of the two systems in terms of the functions $f(t)$ and $g(t)$ make the systems easily amenable to simulation using standard software tools for delay equations including those found within MATLAB.

For the $\sigma<\tau$ situation described in subsection 2.1, we simulate the system of three equations consisting of (2.1), (2.14), and (2.15). Note that one of the delays, $\sigma$, is not explicitly present in this system. However, $\sigma$ plays a role through the formula (2.16), which is used to compute the initial data for the variable $f$ from that for $A$ and $T_{s}$. We measure time in months, so $\tau=12$, corresponding to the maturation time of one year. We chose the birth rate function $B(A)$ to have the form $B(A)=b_{m} A e^{-a A}$, a common choice in the mathematical study of insect infestations, because it reflects the decreasing per capita egg laying rate due to crowding. In this formula the quantity $b_{m}$ is the egg laying rate per female adult beetle per tree at lower densities without the effects of crowding. We assumed that a single female lays on average about 60 eggs during her life (though estimates vary considerably). She is active only in summer, but we average over a year to arrive at a figure of 5 eggs per female per month. Good data on the survival probability for the larvae are not available, but by being deep inside the trees the larvae are well protected from predators, so if we assume a survival probability of about 0.8 , this leads to $\mu_{L}=0.0186$. Values for other parameters are shown in the figures. Figures 1 and 2 illustrate two situations in which $\sigma<\tau$. In Figure 1 the infestation is eradicated, whereas in Figure 2 (in which we used a lower number for the probability $\lambda$ of an infested tree being detected and removed) the number of susceptible trees tends to zero and the entire forest ends up infested, with the number of adult beetles tending to a constant. Other simulations showed a similar outcome but with the number of adults evolving to a periodic cycle. We also noted from our numerical experiments that (2.18) does not appear to be the best possible condition for eradication (i.e., it is sufficient but not necessary).

For the $\sigma>\tau$ situation of subsection 2.2, the appropriate system to simulate is that consisting of (2.21), (2.26), and (2.27). This system involves both delays $\sigma$ and $\tau$ explicitly, with initial data for $g$ calculated from that for $A$ and $T_{s}$ using (2.25) and the initial data formula for $T_{i}$ in (2.12). Figure 3 shows a simulation for the $\sigma>\tau$ situation in which the time between tree infestation and tree removal is a little longer than the time taken for a larva to complete its development and mature as an adult beetle. One expects that it will be more difficult to achieve eradication. The simulation shows that it is still possible to do so, but only by detecting and removing $95 \%$ of infested trees. Figure 4 shows a situation with $\lambda=1$, i.e., every infested tree is later destroyed, but with $\sigma$ chosen to be considerably larger than $\tau$, so that many larvae can complete their maturation and emerge as adults even though their host tree is doomed. With $\lambda=1$, eradication is the final outcome (Theorem 3) even though $\sigma>\tau$, but the simulation shows a large and destructive transient, with very few susceptible trees remaining after the infestation has died out.

4. Discussion. We have derived a mathematical model to describe the infestation of wood boring beetles with the Asian longhorned beetle (ALB) as a prototype. Two delays are needed for the model. One of these delays, denoted by $\sigma$, is the average duration between the time a tree becomes infested and the time the infestation in the tree is detected and the tree removed. The second delay is the maturation delay $\tau$ for the beetle. Since the purpose is to examine whether or not the cut-burn 

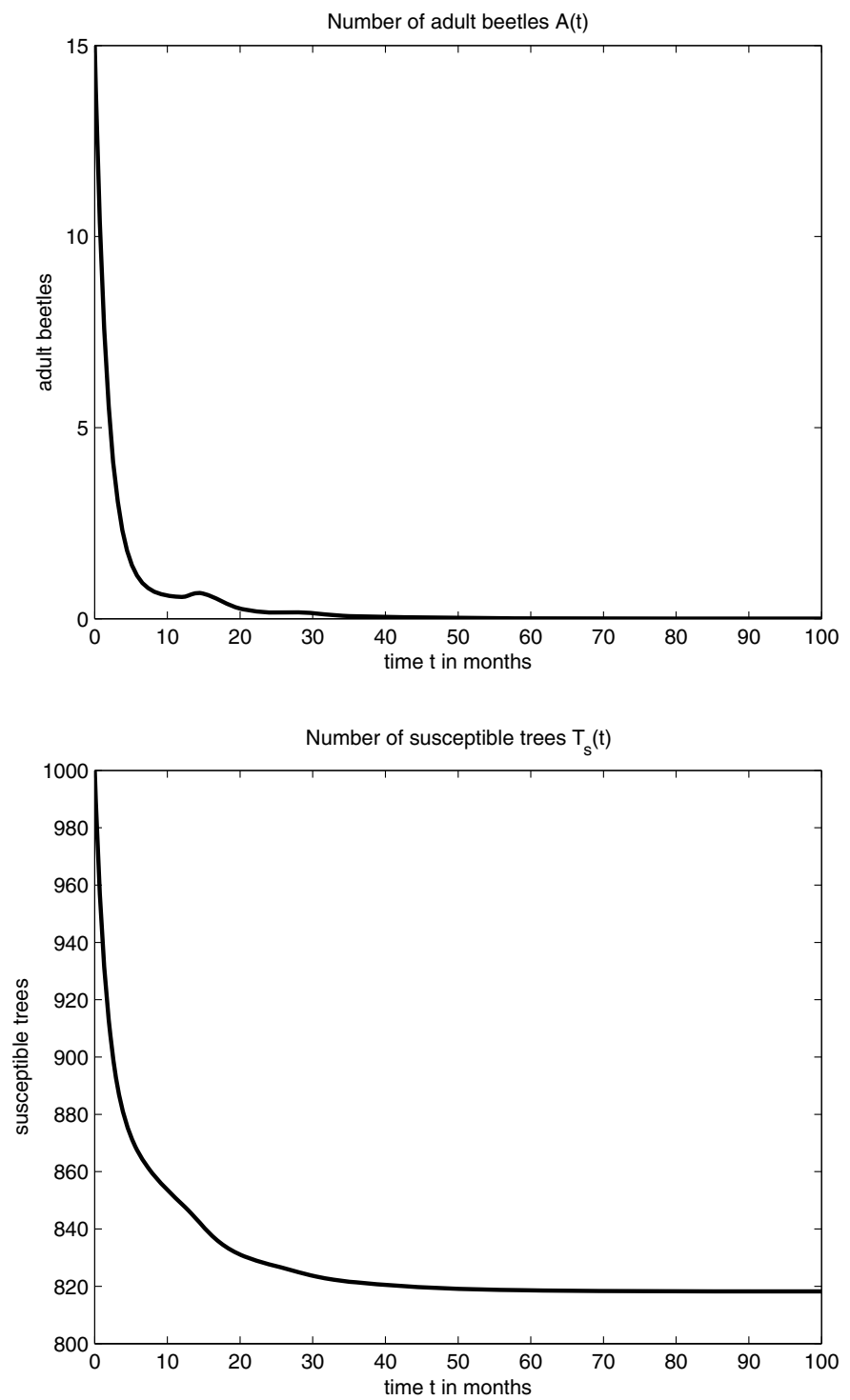

FIG. 1. The $\sigma<\tau$ case: a simulation of (2.1), (2.14), and (2.15). Parameter values were $\tau=12, \lambda=0.9, \beta=0.005, \sigma=3, b_{m}=0.005, a=0.1, \mu_{L}=0.0186, \mu_{A}=0.55$. For these values, (2.18) is satisfied (by a fairly narrow margin) and the infestation is eradicated.

(or removal) control strategy is successful, we have also incorporated a removal rate $\lambda \in[0,1]$ into the model. The parameter $\lambda$ accounts for the possibility that some infested trees might escape detection, which is very likely indeed if ALB activity were to reach wilderness areas, and also permits us to explore the possibility that infestation eradication might be possible without necessarily cutting down and burning every single infested tree. The model assumes different forms depending on whether $\sigma<\tau$ or $\sigma>\tau$, given by (2.1)-(2.4) and (2.21)-(2.24), respectively. 

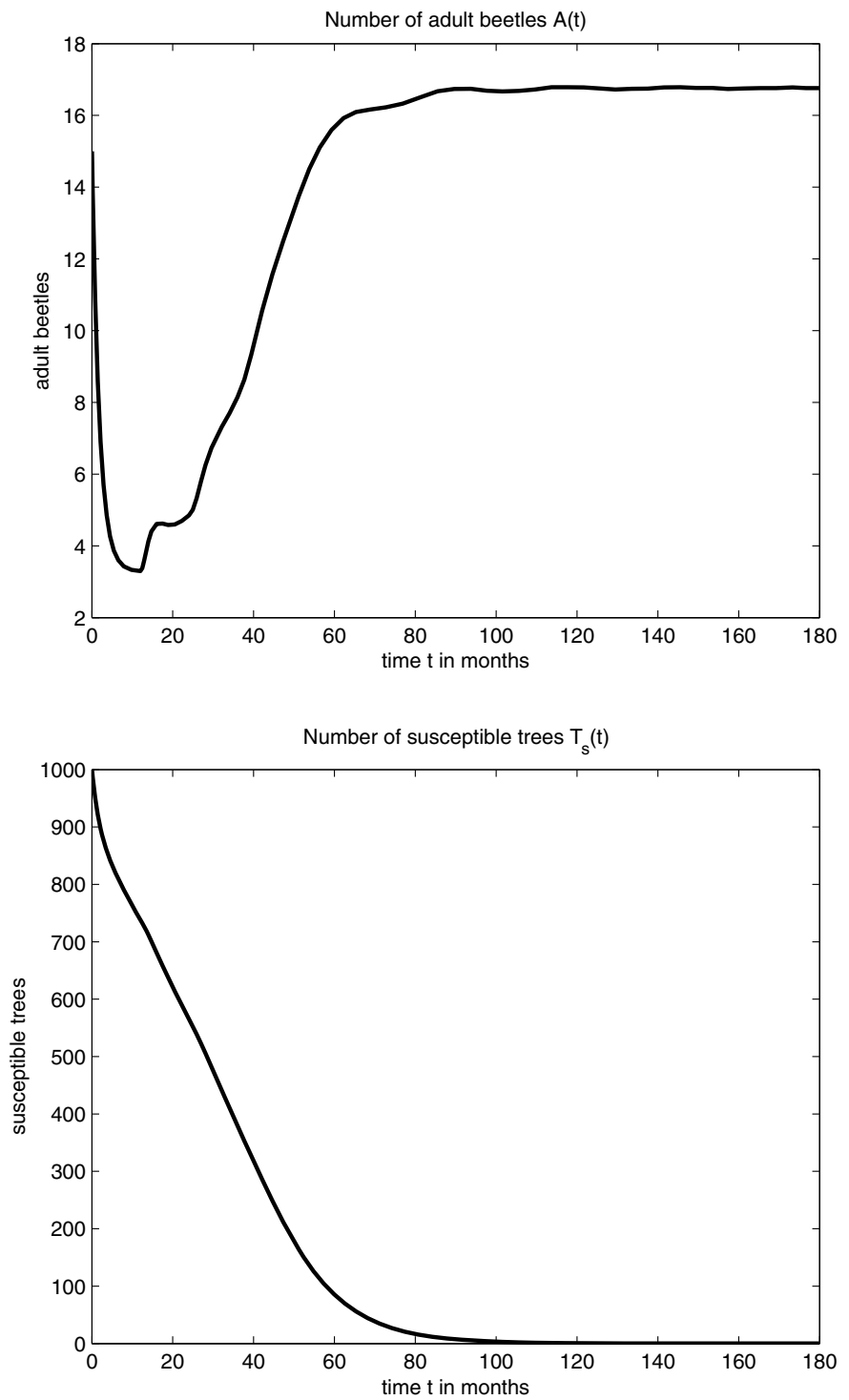

FIG. 2. The $\sigma<\tau$ case: a simulation of (2.1), (2.14), and (2.15). Parameter values were $\tau=12, \lambda=0.4, \beta=0.005, \sigma=3, b_{m}=0.005, a=0.1, \mu_{L}=0.0186, \mu_{A}=0.55$. For these values the infestation takes over the whole forest and the number of adult beetles evolves to a constant.

By applying the comparison method for delay differential equations, we have obtained some conditions for each of the two model systems that are sufficient for infestation eradication. We have also established lower bounds and even approximations for the remaining number of susceptible trees after eradication of the infestation, and this number has economic significance. We have also conducted some numerical simulations which confirm all the theoretical results.

If $\sigma<\tau$, then a beetle larva cannot complete its maturation in a host tree destined for detection and removal. The eradication condition for this case is (2.18), from which 
we conclude that if the proportion $\lambda$ of attacked trees that are detected and removed is sufficiently close to 1 , then the control strategy succeeds. Solving (2.18) for $\lambda$ gives an explicit requirement on $\lambda$. In the case $\sigma>\tau$, detection of infestation in a tree is not happening quickly enough and it may be possible for a beetle larva to complete its maturation even in a tree destined for removal, especially if the larva hatched from an egg that was laid soon after the tree became infested. The corresponding model (2.21)(2.24) is more difficult to analyze, but nevertheless a condition (namely, (2.31)) for eradication of the infestation can be obtained. However, it is more difficult to satisfy the condition. Indeed, if

$$
e^{-\mu_{L} \tau} B^{\prime}(0)\left[\beta \int_{-(\sigma-\tau)}^{0} A(\xi) T_{s}(\xi) d \xi+T_{s}(0)\right]<\mu_{A},
$$

then (2.31) will hold if $\lambda \in[0,1]$ is chosen sufficiently close to 1 . But if (4.1) does not hold, that is,

$$
e^{-\mu_{L} \tau} B^{\prime}(0)\left[\beta \int_{-(\sigma-\tau)}^{0} A(\xi) T_{s}(\xi) d \xi+T_{s}(0)\right] \geq \mu_{A},
$$

then condition (2.31) is never satisfied regardless of the value of $\lambda \in[0,1]$, although we do know from Theorem 3 that the infestation is nevertheless eradicated if $\lambda=1$. To understand the difference between conditions (2.18) and (2.31), note that condition (2.31) relates to the $\sigma>\tau$ situation, in which the timescale for infestation detection in a tree is longer than the maturation time for the beetle. Naturally, we should expect that infestation eradication should be more difficult in the $\sigma>\tau$ situation than in the $\sigma<\tau$ situation in which, if a tree is found to be infested, then its destruction happens sufficiently quickly so that a larva cannot mature in it. In the $\sigma>\tau$ situation it will be possible for some larvae to mature even in a host tree destined for removal.

Note that $\sigma$ and $\lambda$ are the only parameters in the model that are within our control. For example, $\sigma$ could be decreased by the use of high technology acoustic detectors that can detect larval activity in a tree, and $\lambda$, which effectively measures the likelihood of ALB activity being detected in a tree, can be raised by increasing public awareness of the telltale signs of tree infestation. Another related beetle species in Japan has been controlled by the use of fungal bands which contain cultures of insect pathogenic fungi, and there have been trials of the technique on the ALB in Anhui, China (see Hajek et al. [7]). The fungal bands are placed at an approximate height of 2-2.5 m around the trees and infect the adult beetles, which can then transfer the infection during mating, leading to a reduced number of viable eggs. The effectiveness of the technique can be augmented by the use of a chemical attractant. On the use of entomopathogenic nematodes, see also Qin et al. [14].

None of the abovementioned measures is likely to be of much value if ALB were to take hold in wilderness areas. This, of course, highlights the importance of ensuring that the ALB does not become established in North America. Some studies of Keena [8] on the dependence of ALB activity on temperature suggests that the lower 48 states should be able to support beetle survival and reproduction. The numerical simulation work reported in this paper highlights the importance of rapid detection and removal of as many infested trees as possible, and moreover, the simulations demonstrate that if the detection of infestation timescale $\sigma$ is significantly larger than 

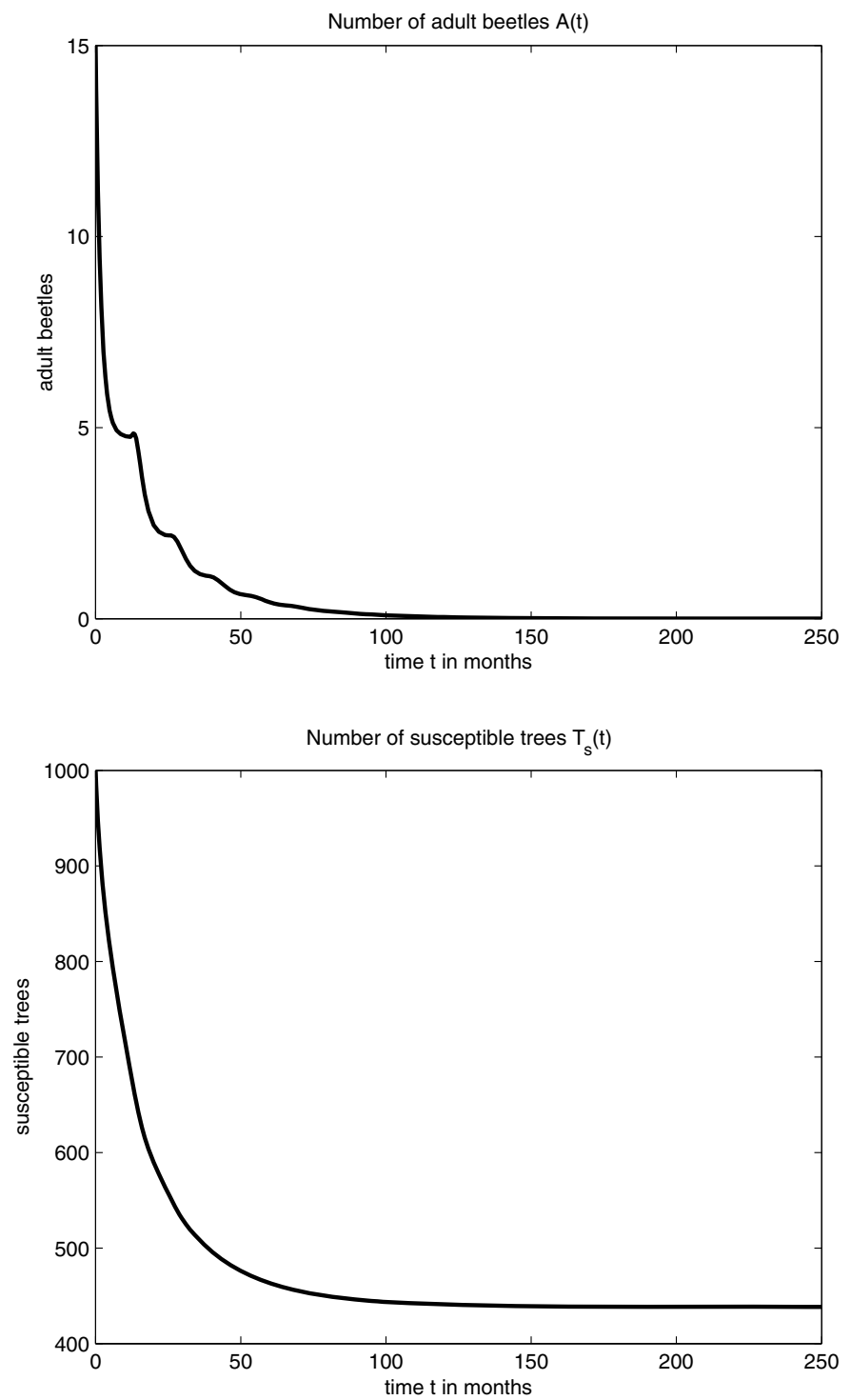

FIG. 3. The $\sigma>\tau$ case: a simulation of (2.21), (2.26), and (2.27). Parameter values were $\tau=12, \lambda=0.95, \beta=0.005, \sigma=14, b_{m}=0.005, a=0.1, \mu_{L}=0.0186, \mu_{A}=0.55$. In this situation it is possible for a larva to complete its maturation in a tree destined for removal. Nevertheless it is possible to achieve eradication, but only with a large proportion of infested trees being detected and removed.

the beetle maturation delay $\tau$, then even though the infestation can be eradicated, the large transients will result in decimation of the forest.

We point out that although the eradication conditions (2.18) and (2.31) are obtained under the hypothesis $B(A) \leq B^{\prime}(0) A$ for $A>0$, this assumption is not crucial. Indeed, if $B(A)$ is continuously differentiable and $B(0)=0$ (which always holds for all birth functions), then by the mean value theorem one can write 

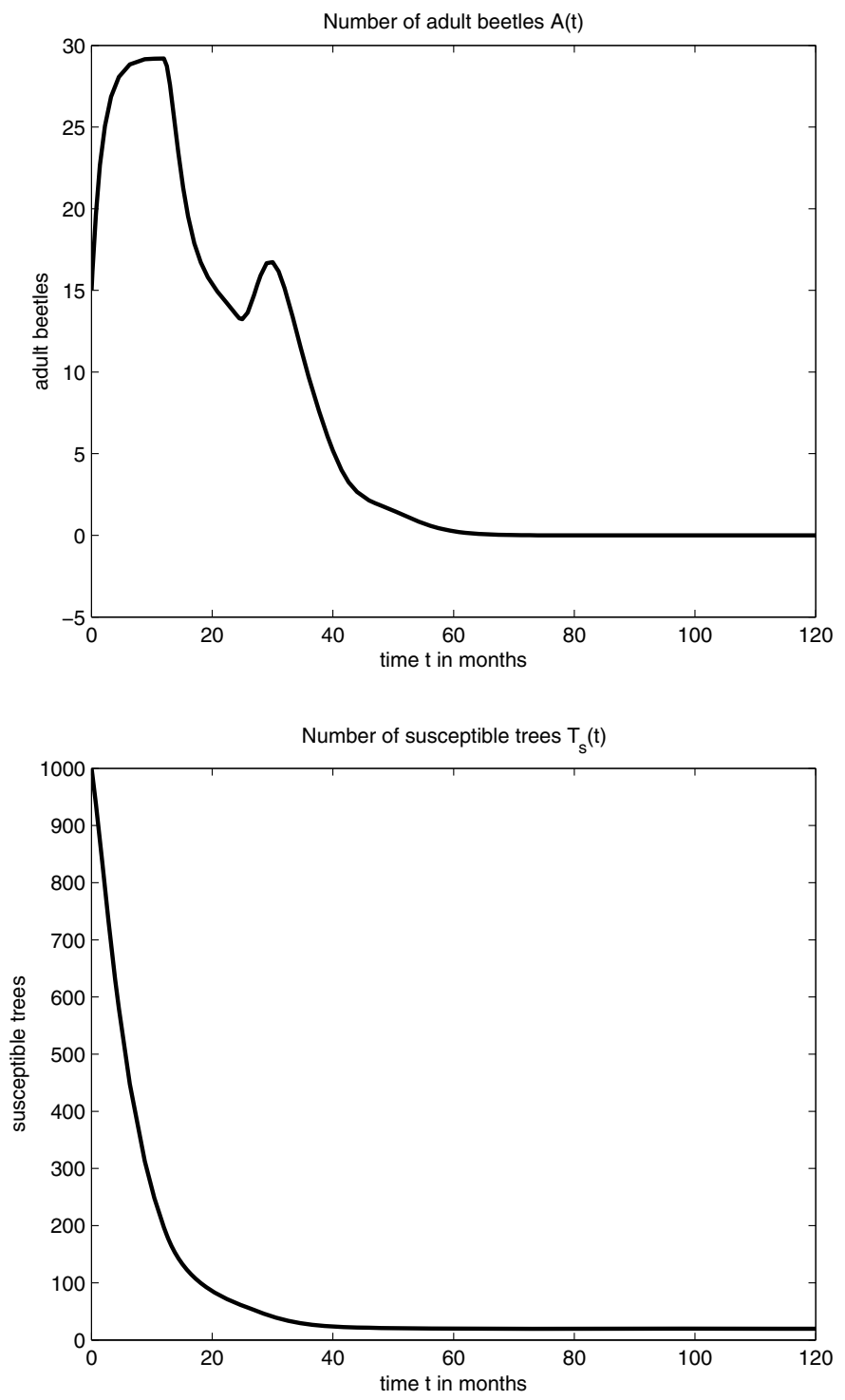

FIG. 4. The $\sigma>\tau$ case: a simulation of (2.21), (2.26), and (2.27). Parameter values were $\tau=12, \lambda=1, \beta=0.005, \sigma=28, b_{m}=0.005, a=0.1, \mu_{L}=0.0186, \mu_{A}=0.55$. In this situation every tree that becomes infested is later destroyed, but with $\sigma$ considerably larger than $\tau$, many larvae can mature and escape as adults before their host tree is destroyed. Nevertheless the final outcome is still eradication, though only after a very destructive transient in which the infestation gets worse. Note that the final number of susceptible trees is very low, indicating severe destruction of the forest during the course of the infestation.

$B(A)=B^{\prime}(\theta) A \leq B_{m} A$, where $B_{m}=\sup _{\theta \geq 0} B^{\prime}(\theta)$. Therefore, the results in Theorems 1 and 2 remain true, with $B^{\prime}(0)$ being replaced by $B_{m}$. Obviously the corresponding eradication conditions become more demanding on $\lambda$.

Spatial spread is another important issue that we have not considered here in this initial work. It is known that the adults of the ALB can fly, but only short 
distances between neighboring trees. Of course, the larvae do not disperse at all. The invasion of the ALB from Asia to North America is believed to have been via the use of wooden packing crates and wood products which may contain individuals in premature stages (eggs, larvae, or pupae). This no doubt accounts for the long range transport between continents and between different districts. Short range dispersal of adult beetles could possibly be modeled, as a first approximation, by the incorporation of Fickian diffusion. However, the actual dispersal behavior of the ALB is not so straightforward, and some experimental work reported in Bancroft and Smith [1] suggests a dependence on beetle density, weather conditions, beetle size and tree size, and that a thorough knowledge of dispersal behavior will be beneficial to eradication efforts. Release of the ALB is prohibited in the US, so there is little experimental work documented, but there have been detailed mark and release studies in Gansu Province, China (Smith et al. [17]) at a site chosen for its landscape similarities to those of urban infestations in the US. These studies suggest that dispersal depends on the spacing of suitable host trees, the age of the beetles, availability of host material, and crowding on suitable trees.

A great deal of mathematical work has been carried out on the dispersal behavior of beetles and insect species more generally (see Shigesada and Kawasaki [15], and Kot, Lewis, and van den Driessche [9]), but not specifically on the ALB. In fact, in our future work on the ALB we are considering the use of integrodifference equations that are continuous in space but discrete in time, since the ALB seems to follow a predictable pattern in nonnative habitats of having one generation per year. Discrete time models have been commonly used in the past to model beetle populations. The flour beetle tribolium has been particularly well studied due to its high rates of reproduction, short life cycle (4 to 6 weeks from egg to adult), ease of culture, and strong cannibalistic tendencies, which make the beetle suitable for laboratory studies (see Costantino and Desharnais [3]). These characteristics do imply that tribolium is very different from the ALB. Cannibalism in particular can affect all pre-adult stages of tribolium, including even some callow (not fully sclerotized) adults (see Mertz [10]).

Costantino et al. [4] compared the results of laboratory studies with the predictions of a discrete time model incorporating, unlike the present study, two pre-adult compartments. In the laboratory studies, adult mortality could be manipulated by removing or adding adults, and recruitment by removing or adding younger adults. Under conditions of high adult mortality, quasi-periodic cycles and chaos were observed in the laboratory populations and in the model predictions.

It seems unlikely that a continuous time model such as the one we present here could work satisfactorily for beetle species which have complex dynamics. Discrete time models might in principle be able to address the issue of complex dynamics, but these models have their limitations too. For example, western pine beetle populations can be asynchronous and indistinguishably overlapped because of differential brood development rates in different trees (see DeMars et al. [5]).

When it comes to modeling the eradication of infestation by removal strategy, we may have to consider diffusion of adults which depends on the removal strength (i.e., the parameter $\lambda \in[0,1])$, since when an infected tree is cut, the adult beetles in that tree will all fly to the neighboring trees. In other words, the removal of infested trees will enhance the diffusion of the adults. This will make modeling a more interesting yet more challenging job. We leave such problems for future investigation.

Acknowledgments. We are grateful to the referees for their very useful comments and suggestions. 


\section{REFERENCES}

[1] J. S. Bancroft And M. T. Smith, Dispersal and influences on movement for Anoplophora glabripennis calculated from individual mark-recapture, Entomologia Experimentalis et Applicata, 116 (2005), pp. 83-92.

[2] C. Castillo-Chavez and H. R. Thieme, Asymptotically autonomous epidemic models, in Mathematical Population Dynamics: Analysis of Heterogeneity, I. Theory of Epidemics, O. Arino et al., eds., Wuerz, Canada, 1995, pp. 33-50.

[3] R. F. Costantino and R. A. Desharnais, Population Dynamics and the Tribolium Model: Genetics and Demography. Springer-Verlag, New York, 1991.

[4] R. F. Costantino, R. A. Desharnais, J. M. Cushing, and B. Dennis, Chaotic dynamics in an insect population, Science, 275 (1997), pp. 389-391.

[5] C. J. Demars, G. W. Slaughter, W. D. Bedard, N. X. Norick, and B. Roettgering, Estimating western pine beetle-caused tree mortality for evaluating an attractive pheromone treatment, J. Chem. Ecol., 6 (1980), pp. 853-866.

[6] R. Gao, X. Qin, D. Chen, And W. Chen, A study on the damage of poplar caused by Anoplophora glabripennis, Forest Research, 6 (1993), pp. 189-193.

[7] A. E. Hajek, B. Huang, T. Dubois, M. T. Smith, and Z. Li, Field studies of control of Anoplophora glabripennis (Coleoptera: Cerambycidae) using fiber bands containing the entomopathogenic fungi Metarhizium anisopliae and Beauveria brongniartii, Biocontrol Science and Technology, 16 (2006), pp. 329-343.

[8] M. A. KeEna, Effects of temperature on Anoplophora glabripennis (Coleoptera: Cerambycidae) adult survival, reproduction, and egg hatch, Environ. Entomol., 35 (2006), pp. 912-921.

[9] M. Kot, M. A. Lewis, and P. van den Driessche, Dispersal data and the spread of invading organisms, Ecology, 77 (1996), pp. 2027-2042.

[10] D. B. Mertz, The tribolium model and the mathematics of population growth, Ann. Rev. Ecol. Syst., 3 (1972), pp. 51-78.

[11] W. D. Morewood, P. R. Neiner, J. C. Sellmer, And K. Hoover, Behavior of adult Anoplophora glabripennis on different tree species under greenhouse condition, Journal of Insect Behavior, 17 (2004), pp. 215-226.

[12] W. D. Morewood, K. Hoover, P. R. Neiner, J. R. McNeil, and J. C. Sellmer, Host tree resistance against the polyphagous wood-boring beetle Anoplophora glabripennis, Entomologia Experimentalis et Applicata, 110 (2004), pp. 79-86.

[13] D. J. Nowak, J. E. Pasek, R. A. Sequeira, D. E. Crane, and V. C. Mastro, Potential effect of Anoplophora glabripennis (Coleoptera: Cerambycidae) on urban trees in the United States, Journal of Economic Entomology, 94 (2001), pp. 116-122.

[14] X. Qin, R. GaO, H. YAng, And G. Zhang, Study on the application of entomopathogenic nematodes, Steinernema bibionis and S. feltiae, to control Anoplophora glabripennis and Holocercus insularis, Forest Science and Research, 1 (1988), pp. 179-185.

[15] N. Shigesada And K. KaWASAKi, Biological Invasions: Theory and Practice, Oxford University Press, Oxford, UK, 1997.

[16] H. L. Smith, Monotone Dynamical Systems. An Introduction to the Theory of Competitive and Cooperative Systems, Math. Surveys Monogr. 41, American Mathematical Society, Providence, RI, 1995.

[17] M. T. Smith, P. C. Tobin, J. Bancroft, G. Li, and R. GaO, Dispersal and spatiotemporal dynamics of asian longhorned beetle (Coleoptera: Cerambycidae) in China, Environ. Entom., 33 (2004), pp. 435-442.

Copyright (C) by SIAM. Unauthorized reproduction of this article is prohibited. 